\title{
Clinical and Histopathological Profile of Subcorneal Pustular Dermatosis
}

\author{
Soumya Venkitaramanan Narayanan ${ }^{1}$, Anitha K. ${ }^{2}$ \\ ${ }^{1}$ Department of Dermatology, P.K. Das Institute of Medical Sciences, Vaniamkulam, Kerala, India. \\ ${ }^{2}$ Department of Dermatology, Government T.D. Medical College, Alappuzha, Kerala, India.
}

\section{ABSTRACT}

\section{BACKGROUND}

Subcorneal Pustular Dermatosis (SPD) is a chronic, benign, recurrent, pustular eruption of idiopathic nature mainly involving the trunk, intertriginous area and flexor aspects of limbs. It is a neutrophilic dermatosis and the exact aetiology is unknown. There are only limited number of studies on the clinical and histopathological aspects of subcorneal pustular dermatosis as well as the associations of subcorneal pustular dermatosis especially in our country. Hence the study is of significance at present.

\section{METHODS}

A descriptive study was done on 37 patients with clinical features of SPD who attended a tertiary care hospital in Kerala during a period of one and a half years. A detailed history, dermatological and system examination, histopathology, immunofluorescence \& relevant laboratory investigations were done, and results were analysed using appropriate biostatistics.

\section{RESULTS}

Majority of the patients were females and belonged to sixth decade. 97.3\% of patients had recurrence of the disease during summer season. Trunk and intertriginous areas were predominantly affected. Hypothyroidism was observed in $21.6 \%$ of patients. Four patients developed chronic plaque psoriasis during the follow up.

\section{CONCLUSIONS}

Subcorneal Pustular Dermatosis is a chronic relapsing pustular eruption which can mimic various other conditions in our daily practice. Many diseases including inflammatory diseases, malignancies and metabolic diseases have been reported to be associated with SPD. Hence a better understanding of the associations and underlying conditions helps in providing effective treatment for the patients.

\section{KEY WORDS}

Subcorneal Pustular Dermatosis, Histopathology, Immunofluorescence, Associations
Corresponding Author: Dr. Soumya Venkitaramanan Narayanan, Assistant Professor, Department of Dermatology,

P. K. Das Medical College,

Kerala, India.

E-mail: soumyamarangad@gmail.com

DOI: $10.14260 /$ jemds/2020/268

Financial or Other Competing Interests: None.

How to Cite This Article:

Narayanan SV, Anitha K. Clinical and Histopathological Profile of Subcorneal Pustular Dermatosis. J. Evolution Med. Dent. Sci. 2020;9(15):1232-1236, DOI: 10.14260/jemds/2020/268

Submission 07-02-2020, Peer Review 20-03-2020,

Acceptance 27-03-2020,

Published 13-04-2020.

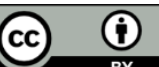




\section{BACKGROUND}

Subcorneal Pustular Dermatosis (SPD) is a rare, chronic, relapsing, sterile pustular eruption characterized by pea sized pustules which are classically described as 'hypopyon' like lesions characterized by half pustular and half clear flaccid blisters. ${ }^{1}$ Presently it is considered as a type of neutrophilic dermatosis. It is histopathologically characterized by subcorneal pustule filled with neutrophils. ${ }^{2}$ SPD was first described by Ian Sneddon and Darrell Wilkinson in 1956. Hence called Sneddon - Wilkinson disease. ${ }^{1}$ SPD is more common in middle aged females between fourth and fifth decade. $(1,3)$ A few studies have reported that rarely SPD can occur in children also.(4,5) A study by Johnson SA et al has reported cases in children as young as 3 months. ${ }^{4}$ Majority of the western studies have not reported any seasonal variation but an study observed that seasonal variation and relapses occurred in 10 patients during successive summer months. ${ }^{6}$

The exact pathogenesis of Subcorneal Pustular Dermatosis is unknown. It is still controversial that whether SPD is a distinct entity or a variant of pustular psoriasis. In one report by Sanchez et al, 10 of 23 patients who were originally diagnosed as SPD developed classic lesions of pustular psoriasis or chronic plaque psoriasis 3-40 years after the onset of SPD. ${ }^{7}$ Various studies reports that there is hyperactivation and migration of neutrophils through the epidermis to aggregate beneath the stratum corneum, leading to subcorneal accumulation of neutrophils in the superficial layers of skin.

Although the exact aetiology of SPD is unknown, previous literature suggest that it is associated with various systemic and other condition. ${ }^{3}$ Connective tissue diseases associated with SPD include rheumatoid arthritis, systemic lupus erythematosus and Sjogren's syndrome. $(8,9)$ SPD is most commonly observed to occur with exacerbation of the connective tissue diseases and has been reported to occur slightly more often in seropositive cases. ${ }^{9}$ Numerous overlap cases of SPD and inflammatory bowel disease have been reported. SPD is more commonly associated with Crohn's disease than ulcerative colitis. ${ }^{10}$ Pyoderma gangrenosum is a neutrophilic dermatosis, which shares many of the disease associations described with SPD particularly monoclonal gammopathies. ${ }^{11}$ Taniguchi et al in 1995 reported SPD in a 48-year-old man with 2-year history of hyperthyroidism. SPD is also associated with hypothyroidism. ${ }^{12}$ The best recognised haematological disorders specifically associated with SPD include monoclonal gammopathies, ${ }^{1}$ multiple myeloma, ${ }^{13}$ aplastic anemia,14 IgG cryoglobulinemia, ${ }^{1}$ lymphomas, 15 chronic lymphocytic leukemia, and chronic myeloid leukemia. ${ }^{14}$ Other rare associations reported include visceral malignancies like apudomas, ${ }^{16}$ drugs like sorafenib, ${ }^{17}$ TNF alpha inhibitors ${ }^{18}$ and Mycoplasma pneumonia infecton. ${ }^{19}$

\section{Clinical Features}

Subcorneal Pustular Dermatosis is a chronic relapsing pustular dermatosis, classically involving flexures that presents abruptly with or without pain and, in some cases, as pruritic superficial papules, which develop into flaccid pustules. Newer lesions appear in the periphery and later coalesce in 24 to 48 hours to form annular, serpiginous or circinate patterns. Most of the subcorneal pustules arise from normal looking skin, yet in some instances, the pustules may appear overlying erythematous or inflamed skin. ${ }^{3}$ These pustules rupture spontaneously to form superficial erosions with crusting that later heals with hyperpigmentation. ${ }^{1}$

\section{Histopathology}

Sneddon and Wilkinson suggest an early histologic examination of lesions, as these will show polymorphonuclear leukocytes migrating from the dermal capillaries to the epidermis. 1 A splitting of the stratum corneal layer from the rest of the epidermis is noted with subcorneal accumulation of neutrophils above a normalappearing dermis, forming a vesiculopustule. The pustules appear to sit on top of the epidermis, usually without forming spongiosis or acantholysis and with minimal change to the epidermis other than the pustules. ${ }^{20,21)}$ Acantholysis may be observed in older lesions of SPD. ${ }^{3}$ Direct and indirect immunofluorescence studies are negative in Subcorneal Pustular Dermatosis which differentiates it from the intraepidermal type of IgA pemphigus. ${ }^{4}$ Subcorneal Pustular Dermatosis can resemble many dermatological diseases both clinically and histopathologically. The main differential diagnosis of SPD includes pustular psoriasis, subcorneal type of IgA pemphigus, pemphigus foliaceus, dermatitis herpetiformis, impetigo and acute generalized exanthematous pustulosis. ${ }^{15}$

\section{Treatment}

The treatment of choice for Subcorneal Pustular Dermatosis is dapsone, but relapses are quite common with discontinuation of the treatment. ${ }^{1}$ Many patients will respond to dapsone and the response to dapsone is also considered as a diagnostic clue to SPD. ${ }^{22}$

\section{METHODS}

After obtaining ethical committee clearance and after getting an informed consent a descriptive study was done on 37 patients (around 2 cases of SPD present to OPD a month. So, sample size was calculated as 36 ) with clinically diagnosed Subcorneal Pustular Dermatosis attending a tertiary care hospital in Kerala, during a period of 18 months. Aim and Objectives were to study the clinical and histopathological profile as well associations of SPD. Those who were on treatment with dapsone, steroids or other Immunosuppressants were excluded from the study. Patients were clinically evaluated with detailed history, complete physical and dermatological examination. Investigations for the known associated factors were done (RBS, TFT, RA factor, pus culture, serum electrophoresis, viral markers [HIV, HBsAg, Anti-HCV]). Skin biopsy was taken from the lesion and was sent for histopathological examination and direct immunofluorescence.

\section{Statistical Analysis}

Data were entered in Microsoft excel sheet \& was analyzed using SPSS (Statistical Programme for Social Science, version 12) software. Qualitative data was analyzed using 
percentages and proportions. Quantitative data was analyzed using mean, standard deviation and median. Association between variables and SPD were checked using Chi-square test.

\section{RESULTS}

A total of 37 patients clinically diagnosed as Subcorneal Pustular Dermatosis were studied during a period of 18 months. Most of the patients (40.5\%) belonged to the age group of $51-60$ years. The mean age of the study population was 56.38 years (Standard Deviation - 8.578). The youngest patient was 39 years old and the oldest was 72 years old. (figure 1). Out of 37 patients studied 3 (8\%) were males and $34(92 \%)$ were females. In the present study 35 patients $(95$ $\%$ ) had exacerbation of disease during summer season. The most common site of lesion was trunk and intertriginous area (81.1\%) followed by lesions over intertriginous area alone $(10.8 \%)$. But none of the patients had lesions involving scalp, palms and soles. Mucosa was also spared in all cases. (Table 1).

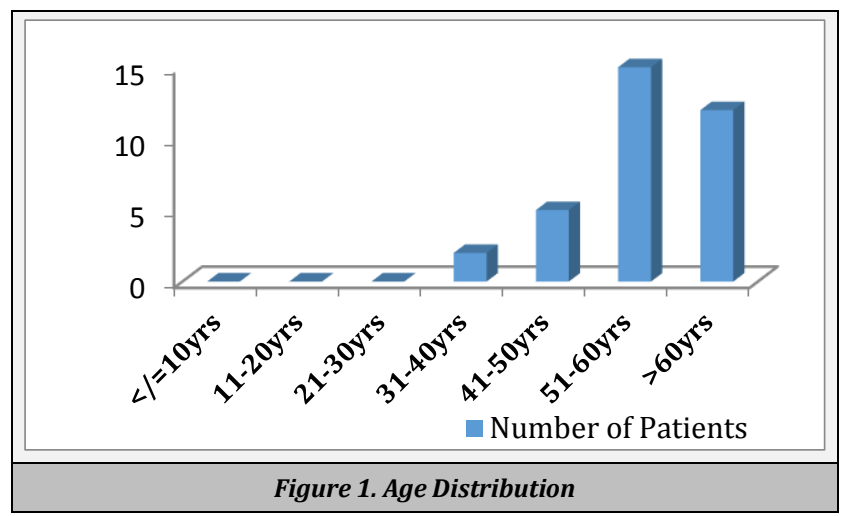

\begin{tabular}{|ccc|}
\hline Site of Lesion & No. of Patients & Percentage \\
Trunk alone & 02 & 5.4 \\
Extremities alone & 01 & 2.7 \\
Intertriginous area alone & 04 & 10.8 \\
Trunk and intertriginous area & 30 & 81.1 \\
Total & $\mathbf{3 7}$ & $\mathbf{1 0 0}$ \\
\hline \multicolumn{2}{c}{ Table 1. Site of Lesion } \\
\hline
\end{tabular}

\begin{tabular}{|ccc|}
\hline Histopathology Features Suggestive of SPD & Present & Absent \\
Subcorneal pustule with neutrophils & 37 & 0 \\
Acantholysis & 2 & 35 \\
Eosinophils & 9 & 28 \\
\hline Table 2. Histopathology Features Suggestive of SPD & \\
\hline
\end{tabular}

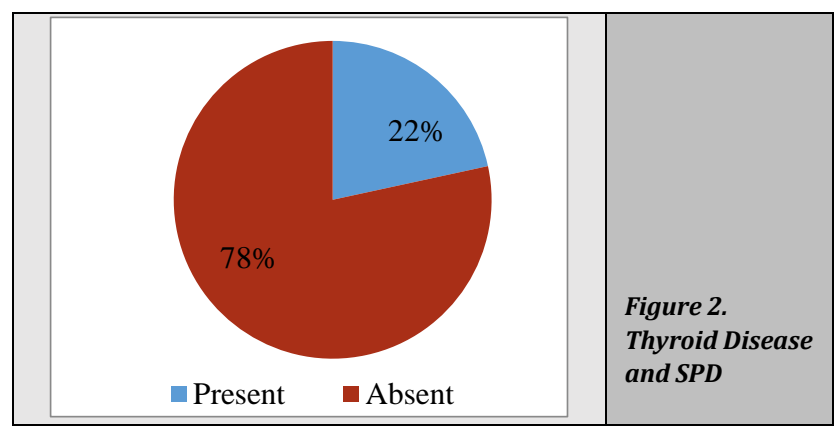

Of our 37 patients, 29 patients (78.4\%) presented with predominantly annular and circinate lesions. Whereas others $(22 \%)$ presented mainly with discrete pustules and few annular lesions. Classical 'Hypopyon' lesions were observed only in one patient. $97.3 \%$ of our patients had history of recurrences. None had family history of psoriasis. Among 37 patients with SPD, 8 (21.6\%) were hypothyroid and none were detected to have hyperthyroidism. This was found to be statistically significant. (Figure 2)

Of the 37 patients with SPD, 2 (5.4\%) patients had Type 2 Diabetes mellitus. Only $1(2.7 \%)$ had connective tissue disease. Culture for fungi and bacteria were negative in 36 out of 37 patients (97.3\%). One culture yielded Staphylococcus aureus. All the 37 clinically diagnosed patients of Subcorneal Pustular Dermatosis showed the characteristic histopathologic features of SPD in the biopsy. Acantholysis was observed in 2 cases and few eosinophils were noted in 9 cases (Table 2). Direct immunofluorescence was negative in all 37 patients. Serum electrophoresis was normal in all our patients. No M band was detected.

Repeat biopsy was done in 4 patients who showed inadequate response to treatment and those who developed features suggestive of chronic plaque psoriasis like scaly plaques over scalp, extensor aspect of extremities and back of trunk. Histopathology revealed epidermal hyperkeratosis and spongiform pustules of Kogoj which were classically suggestive of psoriasis.

\section{DISCUSSION}

Subcorneal Pustular Dermatosis (SPD) or Sneddon Wilkinson's disease is a chronic relapsing pustular eruption, mainly involving the trunk and intertriginous areas which mostly affects the middle-aged women. The exact pathogenesis of SPD is still unknown and its nosological classification is still controversial.

In the present study majority of patients (40.5\%) belonged to the age group of 51 to 60 years. This was in contrast to the previous study by Sneddon and Wilkinson stating that SPD is more common in between fourth and fifth decade. ${ }^{1}$ Our study included 3 males and 34 females with the male to female ratio being 1: 11.3 which is in concordance with previous studies. ${ }^{3}$ But most studies have reported male to female ratio in SPD as 1: $4 . .^{3}$ These contradictory results in age and high prevalence of the disease in females compared to males may be due to the racial differences and varied triggering factors in our population compared to the western world, on whom most of the studies on SPD have been done.

Among the 37 patients in our study, 35 patients (95\%) showed exacerbation of disease during the summer season which is in concordance with an Indian study by Mittal RR ET al. ${ }^{6}$

The site most commonly affected in our study was trunk and intertriginous area (81\%) followed by intertriginous area alone $(10.8 \%)$. The extremities alone were involved in $2.7 \%$. This was in accordance with the previous reports.(1,3) Face and mucosa was never affected in our study. Palm, soles and nails were also spared in all our patients. Annular and circinate lesions were predominantly observed in 29 patients $(78.4 \%)$. The rest of patients presented mainly with discrete pustules along with few annular lesions. The classical 
'hypopyon' lesions of SPD described by Sneddon and Wilkinson was seen only in one patient. ${ }^{1}$

During the period of our study 4 of 37 patients without any family history of psoriasis, who initially presented both clinically and histopathologically as SPD later developed scalp lesions and scaly plaques over trunk suggestive of chronic plaque psoriasis. This is similar to a study by Sanchez et al, but in their study, all the patients who developed chronic plaque psoriasis or pustular psoriasis had family history of psoriasis. ${ }^{7}$

Hypothyroidism was detected in $21.6 \%$ of our patients which is statistically significant. None were detected to have hyperthyroidism in our study. Association of SPD with hypothyroidism and hyperthyroidism has been reported by Taniguchi ET al. ${ }^{12}$

Only 1 of the patients (2.7\%) had rheumatoid arthritis. Butt a et al described 5 cases of SPD associated with rheumatoid arthritis. No other connective tissue diseases were observed among our patients. ${ }^{9}$ Although previous studies report a strong association of SPD with monoclonal gammopathies and multiple myeloma, ${ }^{13}$ electrophoresis doesn't reveal any abnormality among our 37 patients. This may be due to the small sample size.

Culture of 1 among the 37 patients showed growth of Staphylococcus aureus. All others were negative. This was consistent with findings of Sneddon and Wilkinson. ${ }^{1}$ Although there are few anecdotal reports of association of SPD with infections, no significant association was detected in our study. ${ }^{(1,19)}$

All of our 37 patients with clinically diagnosed SPD showed the characteristic histopathological features of Subcorneal Pustular Dermatosis in biopsy. Direct immunofluorescence was negative in all our patients. Biopsy proven cases were treated with dapsone $100 \mathrm{mg}$ daily and most of them responded well to the treatment. But 4 patients $(10.8 \%)$ who responded poorly to treatment and those developed scaly plaques over scalp and trunk were subjected to repeat biopsy which showed features of psoriasis in repeat biopsy which was similar to Sanchez et al study. ${ }^{7}$

\section{CONCLUSIONS}

Subcorneal Pustular Dermatosis is a diagnostic challenge due to its variable presentation. Therefore, it is important to have a thorough clinical and histopathological evaluation of the patients presenting with SPD to avoid misdiagnosis. Many a times SPD like lesions may be an indication of some underlying disorder like gammopathies, thus helping in an early detection. The study of the associations with SPD will also help to control the disease, prevent the recurrence and prolong the remission period by providing appropriate treatment. Moreover, there is a paucity of Indian studies on Subcorneal Pustular Dermatosis. This study thus attains importance in the present settings.

\section{REFERENCES}

[1] Sneddon IB, Wilkinson DS. Subcorneal pustular dermatosis. Br J Dermatol 1956; 68(12):385-94.
[2] Elder D, Elenitsas R, Jaworsky C, et al. Lever's histopathology of the skin. $8^{\text {th }}$ edn. Philadelphia: Lippincott-Raven 1997: p. 238-9.

[3] Naik HB, Cowen EW. Autoinflammatory pustular neutrophilic diseases. Dermatol Clin 2013; 31(3):405-25.

[4] Johnson SA, Cripps DJ. Subcorneal pustular dermatosis in children. Arch Dermatol 1974; 109(1):73-7.

[5] Yayli S, Bahadir S, Alpay K, et al. A case of juvenile subcorneal pustular dermatosis successfully treated with acitretin. Int J Dermatol 2006; 45(9):1131-3.

[6] Mittal RR, Singla A, Gill SS. Subcorneal pustular dermatosis during summer months. Ind J Dermatol Venerol Leprol 1993; 59(6):288-9.

[7] Sanchez NP, Perry HO, Muller SA, et al. Subcorneal pustular dermatosis and pustular psoriasis: a clinicopathologic correlation. Arch Dermatol 1983; 119(9):715-21.

[8] Roger H, Thevenet JP, Souteyrand P, et al. Subcorneal pustular dermatosis associated with rheumatoid arthritis and raised IgA: simultaneous remission of skin and joint involvements with dapsone treatment. Ann Rheum Dis 1990; 49(3):190-1.

[9] Butt A, Burge SM. Sneddon-Wilkinson disease in association with rheumatoid arthritis. Br J Dermatol 1995; 132(2):313-5.

[10] Delaporte E, Colombel JF, Nguyen-Mailfer C, et al. Subcorneal pustular dermatosis in a patient with Crohn's disease. Acta Derm Venereol 1992; 72(4):301-2.

[11] Scerri L, Zaki I, Allen BR. Pyoderma gangrenosum and subcorneal pustular dermatosis, without monoclonal gammopathy. Br J Dermatol 1994; 130(3):398-9.

[12] Taniguchi S, Tsuruta D, Kutsuna $H$, et al. Subcorneal pustular dermatosis in a patient with hyperthyroidism. Dermatology 1995; 190(1):64-6.

[13] Hensley CD, Caughman SW. Neutrophilic dermatoses associated with hematologic disorders. Clin Dermatol 2000; 18(3):355-67.

[14] Park BS, Cho KH, Eun HC, et al. Subcorneal pustular dermatosis in a patient with aplastic anemia. J Am Acad Dermatol 1998; 39(2 Pt 1):287-9.

[15] Ratnarathorn M, Newman J. Subcorneal pustular dermatosis (Sneddon-Wilkinson disease) occurring in association with nodal marginal zone lymphoma: a case report. Dermatol Online J 2008; 14(8):6.

[16] Cheng S, Edmonds E, Ben-Gashir M, et al. Subcorneal pustular dermatosis: 50 years on. Clin Exp Dermatol 2008;33(3):229-33.

[17] Tajiri K, Nakajima T, Kawai K, et al. Sneddon-Wilkinson disease induced by sorafenib in a patient with advanced hepatocellular carcinoma. Intern Med 2015; 54(6):597600.

[18] Sauder MB, Glassman SJ. Palmoplantar subcorneal pustular dermatosis following Adalimumab therapy for rheumatoid arthritis. Int J Dermatol 2013; 52(5):624-8.

[19] Winnock T, Wang J, Suys E, et al. Vesiculopustular eruption associated with Mycoplasma pneumoniae pneumopathy. Dermatology 1996; 192(1):73-4.

[20] Patterson JW. The spongiotic reaction pattern. In: Patterson JW, edr. Weedon's skin pathology. $4^{\text {th }}$ edn. London: Elsevier 2016: p. 103-34. 
[21] Elder D, Elenitsas R, Jaworsky C, et al. Lever's histopathology of the skin. $10^{\text {th }}$ edn. Philadelphia: Lippincott-Raven 2009: p. 267-8.
[22] Macmillan AL, Champion RH. Generalized pustular psoriasis treated with dapsone. Br J Dermatol 1973; 88(2):183-5. 